\title{
An unusual case of accessory head of coracobrachialis muscle involving lateral cord of brachial plexus and its clinical significance
}

\author{
S.A. Garbelotti Jr. ${ }^{1}$, S.R. Marques ${ }^{1}$, P.R. Rocha1 ${ }^{1}$, V.R. Pereira ${ }^{2}$, L.O. Carvalho de Moraes ${ }^{1}$ \\ ${ }^{1}$ Disciplina de Anatomia Descritiva e Topográfica, Departamento de Morfologia, Universidade Federal \\ e São Paulo - Escola Paulista de Medicina, São Paulo, Brasil \\ ${ }^{2}$ Laboratório de Anatomia Humana do Centro Universitário São Camilo, São Paulo, Brasil
}

[Received: 10 January 2017; Accepted: 23 March 2017]

\begin{abstract}
Knowledge of anatomical variations in the peripheral nervous system is key in the interpretation of unusual clinical signs or during physical or diagnostic imaging. This case study is a description of an anatomical variation between the coracobrachialis muscle and brachial plexus. In a routine dissection in the human anatomy laboratory, we were faced with an anatomical variation in the coracobrachialis muscle, observed in the upper right limb of a male cadaver. The coracobrachialis muscle had a common origin at the apex of the coracoid process and then divided into two heads. The lateral head followed its normal course until insertion into the middle third of the humerus, while the medial head involved the lateral cord of the brachial plexus before insertion into the intermuscular septum in the proximal third of the humerus. Atypical anatomical variations have clinical and surgical implications in procedures such as brachial plexus block and lateral cord compression. In these cases the result could be paralysis of the flexor musculature of the forearm and hypoesthesia of the forearm. (Folia Morphol 2017; 76, 4: 762-765)
\end{abstract}

Key words: coracobrachialis muscle, brachial plexus, anatomical variation, anaesthetic blockade of the brachial plexus

\section{INTRODUCTION}

The coracobrachialis muscle is part of the second muscular layer in the anterior region of the arm. It has the coracoid process as the proximal insertion, in common with the short head of the biceps brachii muscle. It is inserted distally into the medial margin of the humeral shaft, between the distal insertions of the brachialis and triceps muscles. In a regular syntopy, the coracobrachialis muscle is pierced and innervated by the musculocutaneous nerve (MN) [9]; however, in some cases it has been reported that the MN does not pierce the coracobrachialis muscle [6].
The coracobrachialis, pectoralis major, short head of the biceps brachii, clavicular fibres of the deltoid, and subscapularis muscles form an effective muscle group for flexion and adduction of the arm, in addition to avoiding anterior dislocation of the shoulder $[1,6]$.

From a morphological and surgical standpoint, the coracobrachialis muscle plays an important role in post-mastectomy reconstruction, infraclavicular and axillary deformities, and muscle transfer for the treatment of paralysis, as well as being a guide for axillary artery access and anaesthetic blockade of the brachial plexus $[6,7]$.

Address for correspondence: S.A. Garbelotti Jr, PhD, Disciplina de Anatomia Descritiva e Topográfica, Departamento de Morfologia e Genética Edifício Leitão da Cunha, Universidade Federal de São Paulo - Escola Paulista de Medicina, Rua Botucatu, 740 - Vila Clementino - 04023-900, São Paulo, SP, Brasil, tel: 55-11-5576-4848 voip. 2228, fax: 55-11-5571-7597, e-mail: silviogarbelotti@gmail.com 


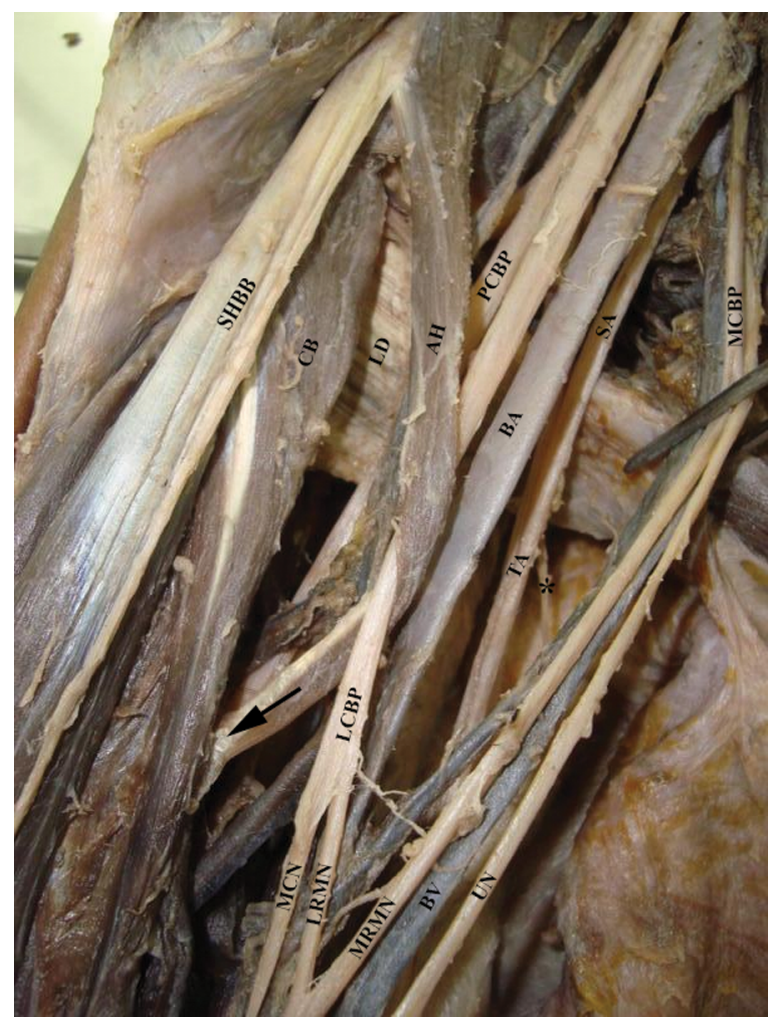

Figure 1. Anterior view of the axilla showing the short head of the biceps brachii muscle (SHBB), the coracobrachialis muscle (CB), the accessory head (AH) involving the lateral cord of the brachial plexus (LCBP), and inserting proximally in the anteromedial humerus surface (arrow). The division of the lateral cord that originated at the lateral root of the median nerve (LRMN), the musculocutaneous nerve (MCN). Medially to the accessory head, brachial artery (BA) and medial cord of the brachial plexus (MCBP) that divides into the ulnar nerve (UN) and medial root of the median nerve (MRMN); between them the basilica vein (BV). Deeply, the insertion of the latissimus dorsi aponeurosis (LD), posterior cord of the brachial plexus (PCBP) and the subscapular artery (SA) dividing into the thoracodorsal (TA) and circumflex scapular arteries (asterisk).

Knowledge of anatomical variations in the peripheral nervous system is key in the interpretation of unusual clinical signs or during physical or diagnostic imaging. This study aims to point out an unusual anatomical variation between the coracobrachialis muscle and lateral cord of the brachial plexus, providing important information to clinicians, surgeons, and physiotherapists who perform procedures in this region [5-7].

\section{CASE REPORT}

In a routine dissection in the human anatomy laboratory, we found an anatomical variation in the coracobrachialis muscle observed in the right upper limb of a male cadaver of 53 years of age. The muscle

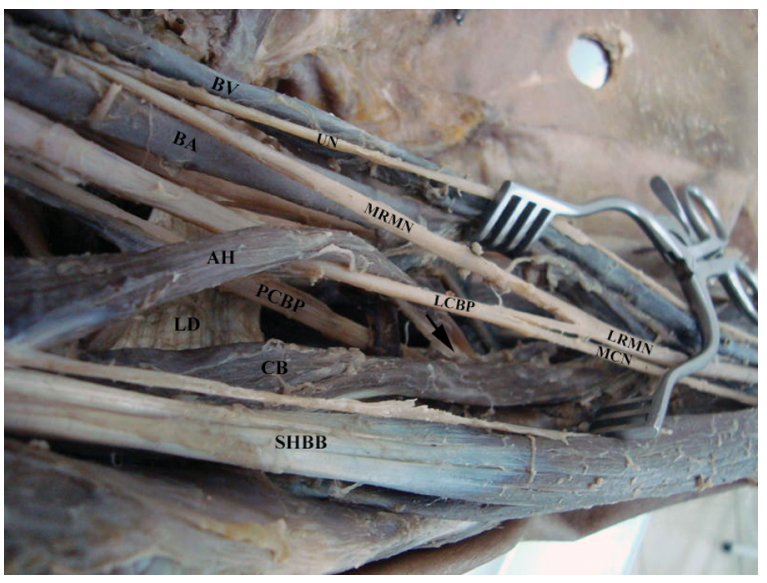

Figure 2. Superolateral view showing the short head of the biceps brachii muscle (SHBB), the coracobrachialis muscle (CB) and the accessory head $(\mathrm{AH})$ of coracobrachialis muscle involving the lateral cord of the brachial plexus (LCBP) and the distal formation of the musculocutaneous nerve (MCN) not pierce the coracobrachialis muscle. The division of the lateral cord that originated at the lateral root of the median nerve (LRMN), the musculocutaneous nerve. Medially to the accessory head, the ulnar nerve (UN) and the medial root of the median nerve (MRMN) show up between the brachial artery (BA) and basilica vein (BV). Deeply, the insertion of the latissimus dorsi aponeurosis (LD), the posterior cord of the brachial plexus (PCBP).

had proximal insertion at the apex of the coracoid process, dividing after $5.89 \mathrm{~cm}$ into two heads.

The lateral head followed its normal course until insertion into the middle third of the humerus. The accessory head involved the lateral cord of the brachial plexus at $11.64 \mathrm{~cm}$ from the coracoid process before insertion into the intermuscular septum in the proximal third of the humerus, approximately $1.5 \mathrm{~cm}$ distal to the insertion of the latissimus dorsi muscle.

The division of the lateral cord originated at the lateral root of the median nerve and the MN began $17.3 \mathrm{~cm}$ from the coracoid process. The MN did not pierce the coracobrachialis muscle (Figs. 1, 2).

The innervation of the coracobrachialis muscle and accessory head occurred directly from the lateral cord of the brachial plexus and the blood supply originated from the brachial artery branch.

\section{DISCUSSION}

Embryologically, the presence of anatomical neuromuscular variations can be attributed to random factors that influence the mechanism of limb muscle formation. Factors directing nerve growth are chemo- 
attractive and repellent that direct cellular processes to proper tissue formation.

During the development period, the lateral plate of the mesoderm gives rise to limb buds and the mesenchyme of these buds differentiate into the deeper structures of the limbs, while the axons of the peripheral nerves grow distally from the ectoderm to reach the muscles and skin [3].

Butz et al. [2] state that signalling mechanisms during embryological development might play a role during the fifth week of development, axons of the spinal nerves grow distally to initiate contact with the mesenchyme of the limb, and inadequate signalling may negatively influence the normal formation of the plexus.

At one point during development, a single muscle mass is formed by fusion of the primary muscle into the different layers of the arm which then disappears through apoptosis. Failure in this process of disappearance of the primary muscle during embryonic development may have resulted in the presence of an anomalous coracobrachialis muscle [5]. This embryological explanation is in line with what we believe to have happened in our finding.

Variations in the coracobrachialis muscle are already well described in the literature; however, the presence of the accessory head coracobrachialis muscle is an unusual anatomical variation and was first described by Wood in 1867 [see 10], who reported that the muscle had a proximal origin in the coracoid process and a distal insertion in the surgical neck of the humerus.

Other variations have been described, the most common being the anatomical variation in the origin of the median and MNs, in which the lateral cord of the brachial plexus pierced the coracobrachialis muscle and then bifurcated in the middle third of the arm into two terminal branches originating the median and MNs [1, 2]. Our finding differs from these studies as the lateral cord did not pierce the coracobrachialis muscle but involved it, and then bifurcated inferiorly into two terminal branches, the median and MNs.

El-Naggar and Zahir [4] described the anatomical variation in the coracobrachialis muscle in the left upper limb of an adult cadaver that presented two muscular heads formed inferiorly to its proximal insertion, having its distal insertions in the middle of the anteromedial humeral surface and in the aponeurosis of the triceps medial head. The authors suggested that the two coracobrachialis muscle heads might represent the incomplete fused heads of the primordial muscle.

El-Naggar and Al-Saggaf [3] and Potu et al. [8] reported another kind of coracobrachialis variation where the distal muscle insertions occurred in the middle of the anteromedial humerus surface and in an aponeurotic arch shape expansion that fixed in the lateral epicondyle. In this case, as the median nerve and deep brachial artery passed down the arch, there was a possibility of neurovascular compression.

Our finding is not similar to these cases, which gives it a high degree of interest, as in the anatomical variations found the innervation of the coracobrachialis muscle and accessory head occurred in the brachial plexus before muscular innervation [4].

Gupta et al. [5] report that anatomical variations are rare where the lateral cord pierces the coracobrachialis muscle and then bifurcates in the MN and lateral branch of the median nerve.

The coracobrachialis muscle plays an important role as a guide in surgical procedures and anaesthetic blockade of the brachial plexus and has been suggested in reconstructions of deformities due to mastectomy. In addition to having its tendon shift in surgery for recurrent anterior dislocation of the shoulder, providing dynamic stabilisation of the glenohumeral joint, especially when the arm is in a vulnerable position of abduction and lateral rotation $[1,6,7]$.

\section{CONCLUSIONS}

The important neuromuscular anatomical variation reported advises clinical and surgical professionals that recognition of anomalous muscles and their unusual peripheral neural connections are extremely relevant for both the differential diagnosis of incomprehensible symptoms and for performing local procedures such as nerve block, image diagnosis, or surgical procedures.

\section{REFERENCES}

1. Abhaya A, Khanna J, Prakash R. Variation of the lateral cord of brachial plexus piercing coracobrachialis muscle. Ann Soc India. 2003; 52(2): 168-170.

2. Butz JJ, Shiwlochan DG, Brown KC, et al. Bilateral variations of brachial plexus involving the median nerve and lateral cord: An anatomical case study with clinical impli- 
cations. Austr Med J. 2014; 7(5): 227-231, doi: 10.4066/ AMJ.2014.2070, indexed in Pubmed: 24944720.

3. El-Naggar MM, Al-Saggaf S. Variant of the coracobrachialis muscle with a tunnel for the median nerve and brachial artery. Clin Anat. 2004; 17(2): 139-143, doi: 10.1002/ ca.10213, indexed in Pubmed: 14974102.

4. El-Naggar MM, Zahir FI. Two bellies of the coracobrachialis muscle associated with a third head of the biceps brachii muscle. Clin Anat. 2001; 14(5): 379-382, doi: 10.1002/ ca.1067, indexed in Pubmed: 11754228.

5. Gupta G, Singh K, Chhabra S, et al. Accessory coracobrachialis: a case report with its morphological and clinical significance. Surg Radiol Anat. 2012; 34(7): 655-659, doi: 10.1007/s00276-011-0931-6, indexed in Pubmed: 22314797.

6. Ilayperuma I, Nanayakkara BG, Hasan R, et al. Coracobrachialis muscle: morphology, morphometry and gender differences. Surg Radiol Anat. 2016; 38(3): 335-340, doi: 10.1007/s00276-015-1564-y, indexed in Pubmed: 26464302.

7. Kopuz C, Içten N, Yildirim M. A rare accessory coracobrachialis muscle: a review of the literature. Surg Radiol Anat. 2003; 24(6): 406-410, doi:10.1007/s00276-002-0079-5, indexed in Pubmed: 12652369.

8. Potu BK, Rao MS, Nayak SR, et al. Variant insertion of coracobrachialis muscle in a South Karnataka cadaver. Cases J. 2008; 1(1): 291, doi: 10.1186/1757-1626-1-291, indexed in Pubmed: 18976467.

9. Standring S. Gray's anatomy. 41st Ed. Elsevier, Churchill Livingstone 2016.

10. Sugalski MT, Wiater JM, Bigliani LU, et al. Coracobrachialis brevis: Anatomic anomaly. J Shoulder Elbow Surg. 2003; 12(3): 306-307, doi: 10.1016/mse.2003. S1058274603000466, indexed in Pubmed: 12851588. 